\title{
Preventing the Establishment of a Wildlife Disease Reservoir: A Case Study of Bovine Tuberculosis in Wild Deer in Minnesota, USA
}

\author{
Michelle Carstensen ${ }^{1}$ and Michael W. DonCarlos \\ ${ }^{1}$ Minnesota Department of Natural Resources, Wildlife Health Program, 5463-C West Broadway, Forest Lake, MN 55025, USA \\ ${ }^{2}$ Minnesota Department of Natural Resources, Wildlife Research and Policy, 500 Lafayette Road, St. Paul, MN 55155, USA
}

Correspondence should be addressed to Michelle Carstensen, michelle.carstensen@state.mn.us

Received 14 January 2011; Accepted 4 March 2011

Academic Editor: Mitchell V. Palmer

Copyright (C) 2011 M. Carstensen and M. W. DonCarlos. This is an open access article distributed under the Creative Commons Attribution License, which permits unrestricted use, distribution, and reproduction in any medium, provided the original work is properly cited.

\begin{abstract}
Bovine tuberculosis (bTB) has been found in 12 cattle operations and 27 free-ranging white-tailed deer (Odocoileus virginianus) in northwestern Minnesota, following the state's most recent outbreak of the disease in 2005 in the northwest part of the state. Both deer and cattle have the same strain of bTB. The Minnesota Board of Animal Health has been leading efforts to eradicate the disease in Minnesota's cattle, which have included the depopulation of all infected herds, a cattle buy-out program, and mandatory fencing of stored feeds. The Minnesota Department of Natural Resources began surveillance efforts in free-ranging white-tailed deer in fall 2005. All bTB-infected deer have been found within a $16 \mathrm{~km}^{2}$ area in direct association with infected cattle farms. Aggressive efforts to reduce deer densities through liberalized hunting and sharpshooting have resulted in a 55\% decline in deer densities. Also, recreational feeding of wild deer has been banned. Disease prevalence in deer has decreased from $1.2 \%$ in 2005 to an undetectable level in 2010.
\end{abstract}

\section{Introduction}

Bovine tuberculosis (bTB), caused by Mycobacterium bovis, is a chronic infectious disease that affects a wide range of mammals, including domestic cattle and humans [1]. Because of the serious economic implications and zoonotic concerns associated with bTB, efforts to eradicate this disease internationally have been extensive. Wildlife species can play an important role in the epidemiology of bTB, but it is important to distinguish between spillover and maintenance wildlife hosts. A spillover host needs continuing exposure to the bacterium from other species to maintain infection; whereas maintenance hosts can maintain infection without cross-transmission from other species of domestic or wild animals [1]. While both spillover and maintenance hosts may act as a disease vector, a true bTB wildlife reservoir with epidemiological implications for disease control requires that the maintenance host have the potential to transmit the pathogen to other species [2, 3]. Examples of wildlife reservoirs for $M$. bovis exist in various regions of the world, including African buffalo (Syncerus caffer, South Africa), wood bison (B. bison athabascae, Canada), European badger (Meles meles, United Kingdom), brushtail possum (Trichosurus vulpecula, New Zealand), European wild boar (Sus scrofa, Spain), elk (Cervus elaphus, Canada), and white-tailed deer (Odocoileus virginianus, United States) [3-8]. These wildlife reservoirs share some common characteristics, such as high population densities, artificial feeding, and continuous interactions at the wildlife-livestock interface, which perpetuate the disease and make bTB control efforts especially challenging. However, it may be possible to prevent the establishment of a wildlife reservoir if a spillover event can be contained, thus allowing domestic animal disease control efforts to take effect.

Bovine tuberculosis had been eradicated in Minnesota's cattle industry in 1971; however, the disease reemerged in the 
state in 2005. A beef cow with thoracic lesions was discovered through routine slaughter surveillance at a slaughter facility in Wisconsin in July 2005. This cow was traced to a beef herd in northwest Minnesota; subsequent testing revealed $1.2 \%$ bTB prevalence in the herd [9]. Epidemiological investigation of trace-in and trace-out cattle movements and area herd-testing led to the detection of 4 additional bTB-positive cattle herds in the region by October 2005. All bTB-infected cattle herds were appraised, indemnified, and depopulated by the United States Department of Agriculture (USDA). Strain-typing concluded the M. bovis had southwestern U.S. or Mexican origin and was distinctly different than strains of M. bovis in Michigan and Manitoba [10]. In November 2005, the Minnesota Department of Natural Resource (MNDNR) sampled 474 hunter-harvested deer for bTB within $25 \mathrm{~km}$ of the infected cattle farms. One deer, harvested within 1 mile of the index cattle herd, was found infected with bTB (apparent prevalence $0.2 \%$ ); strain-typing concluded that it was the same bTB strain as the cattle [11]. Deer shooting permits were issued to landowners of bTB-infected farms in January 2006, resulting in 90 additional deer being harvested, including a second bTB-positive deer. Because both bTBinfected deer were harvested in direct association with infected farms, deer shared the same strain of the disease as cattle, and no positive deer were detected in the larger area of sampling, we postulated that the wildlife infections were a result of direct spillover from bTB-positive cattle.

In 2006, surveillance efforts were intensified for both cattle and deer in northwest Minnesota, and a one-time statewide sampling effort that tested 1,554 cattle herds and 4,000 deer was conducted at the request of USDA. No bTB infection was detected outside the 4-county region of northwest Minnesota in deer or cattle. However, new cases of bTB were discovered in 2 cattle herds and 5 deer in the northwest, prompting the establishment of a Bovine Tuberculosis Management Zone as well as an inner target area, called the Bovine TB Core Area. The establishment of these control zones enabled more focused disease management efforts in deer, and the same boundaries were later adopted by the Minnesota Board of Animal Health (BAH) for disease management efforts in cattle that included increased testing requirements and movement restrictions. By the end of 2006, Minnesota had lost its Bovine TB-Free accreditation, and the entire state was demoted to Modified Accredited Advanced status [12]. This initial loss of bTB-Free accreditation prompted political and public support for aggressive disease control management, as increased cattle testing requirements and trade restrictions had major economic consequences for the entire state's cattle industry.

The MNDNR began its aggressive disease management campaign to eradicate bTB in deer by instituting a ban on recreational feeding of wild cervids in a $10,060-\mathrm{km}^{2}$ area of northwest Minnesota in November 2006; baiting had already been outlawed statewide since 1991. As direct or indirect transmission of $M$. bovis between wild hosts or livestock can occur at shared feed sources, restricting supplemental feeding of wild cervids can limit disease propagation [13]. Efforts to dramatically reduce deer densities in the region followed, with emphasis on the Bovine TB Core Area. During winter
2007, agency-sponsored sharpshooting of deer within the Bovine TB Core area began as an effort to further minimize disease spread outside the area by reducing deer densities and removing potentially bTB-infected individuals. Over 4 consecutive winters (2007-2010), approximately 2,600 deer were removed by ground and aerial sharpshooting in the Bovine TB Core area, including 14 bTB-positive deer. Liberalized hunting opportunities were initiated by fall 2007. This included the creation of a special deer management unit that encompassed the Bovine TB Management Area, early and late season hunts, and reduced-cost bonus permits. Landowners living within the Bovine TB Management Area were issued shooting permits to harvest deer on private lands outside the bounds of traditional deer harvest seasons.

On the cattle side of the bTB issue, the detection of 4 new bTB-infected herds in the northwest in 2007 resulted in a further loss of the state's bTB Modified Accredited Advanced status to Modified Accredited. This change fueled a political campaign that facilitated the Minnesota State Legislature to establish and fund a voluntary cattle buy-out program. By Jan 2009, 46 farms accepted the buy-out program and 6,200 cattle were removed from the Bovine TB Management Zone. Remaining farms were required to fence stored feed and winter feeding areas; the state providing funding for fencing at a 90:10 cost share with producers up to $\$ 75,000$. One additional bTB-infected cattle herd was discovered during the buy-out program, bringing the total number of bTBinfected farms detected since 2005 to 12 . Minnesota was granted a split-state status by USDA in 2008, which upgraded its status to Modified Accredited Advanced through most of the state, with only a $6,915-\mathrm{km}^{2}$ area in the northwest remaining Modified Accredited. With the last bTB-infected cattle herd being discovered in January 2009, Minnesota regained TB-Free accreditation in October 2010 in the majority of the state, with only the split-state region of the northwest remaining Modified Accredited Advanced.

Since the bTB surveillance began in deer, 27 infected deer have been discovered. All of these bTB-infected deer were harvested within $16 \mathrm{~km}$ of the first bTB-infected cattle herd. The last bTB-infected deer was killed in November 2009. Sharpshooting during winter 2010 in the Bovine TB Core area failed to find additional infected deer, which marked the first time that intensive culling had occurred in this area without finding bTB-positive deer. Recent hunter-harvested surveillance in fall 2010 also failed to detect any obvious cases of the disease, although final test results are pending.

Declining trends in bTB prevalence in deer and the limited geographic extent of infected individuals lends optimism that this disease may have been eradicated in the local deer herd or remains present at an undetectable level. Further, nearly all (96\%) of the bTB-infected deer were older animals, born during or before 2005. To call attention to this skewed age distribution of bTB-positive deer, we coined the phrase "Alive in '05;" meaning all infected deer were born on or prior to 2005, when the disease was first discovered in the region. The apparent absence of bTB infection in younger age classes further suggested that this disease was not maintaining itself within the local deer population, but continued monitoring is required to confirm 
the absence of bTB in the region. Consequently, the state will continue hunter-harvested surveillance of deer for bTB at least through 2014.

Minnesota's primary goal has been the eradication of bTB from both deer and cattle. The aim of this paper is to describe the primary management strategies implemented by MNDNR to prevent the establishment of a wildlife disease reservoir in free-ranging white-tailed deer. These strategies included, (1) rapid response to initial disease detection, (2) follow-through on monitoring the outbreak with adequate surveillance, (3) recognizing when monitoring must switch to management, (4) aggressively reducing transmission potential by reducing deer densities, limiting recreational feeding and mitigating risks at the cattle-wildlife interface, and (5) evaluation of efforts and adjusting as needed.

\section{Materials and Methods}

2.1. Bovine Tuberculosis Surveillance Areas. There were 3 primary surveillance areas used to monitor the bTB outbreak and focus disease management efforts. These included a (1) Split-state Zone, which was established in 2008 by USDA for disease control efforts for cattle, (2) Bovine TB Management Zone, which was established by MNDNR in 2006 and lies within the Split-state Zone, and (3) Bovine TB Core Area, also established by MNDNR in 2006 as a focal area within the Bovine TB Management Zone (Figure 1).

Minnesota's $3,884 \mathrm{~km}^{2}$-Split-state Zone is located between $48^{\circ} 11^{\prime} \mathrm{N}$ and $49^{\circ} 0^{\prime} \mathrm{N}$ latitude and $94^{\circ} 56^{\prime} \mathrm{W}$ and $95^{\circ} 58^{\prime} \mathrm{W}$ longitude in northwest Minnesota. There were an estimated 300 cattle, 2 bison, 7 goat, and 1 captive cervid (white-tailed deer) herds in the zone [14]. Of the cattle herds, 19 were dairy operations with $<200$ animals per farm. The remaining cattle farms were beef cow/calf operations that averaged 80 animals per herd. Approximately 53\% of the zone was publically owned by state, federal, county and tribal authorities. Private land accounted for about $3,280 \mathrm{~km}^{2}$.

The $1,567 \mathrm{~km}^{2}$-Bovine TB Management Zone is located between $48^{\circ} 18^{\prime} \mathrm{N}$ and $48^{\circ} 41^{\prime} \mathrm{N}$ latitude and $95^{\circ} 15^{\prime} \mathrm{W}$ and $95^{\circ} 49^{\prime} \mathrm{W}$ longitude in northwest Minnesota. Its boundaries were defined by delineating a $16-\mathrm{km}$ buffer around the 7 bTB-infected deer discovered by fall 2006. The landscape was comprised of 25\% flat, mixed forest, 35\% lowland marsh, and $40 \%$ open agriculture. Land ownership was approximately $57 \%$ public (state-owned), $40 \%$ private, and $3 \%$ tribal. Sixty-six cattle farms existed in the zone prior to the 2008 buy-out program, which removed 6,200 cattle from 46 herds. Twenty herds remained with approximately 1,500 cattle. Three small captive cervid operations also existed in the zone. Prefawning deer densities ranged from 2.3 to 3.1 deer $/ \mathrm{km}^{2}$, with reduced densities in the bTB Core Area as described below. A small, reintroduced elk (Cervus elaphus) population numbering 40-60 animals ranged into the southern half of the zone. From 2004-2009, 77 wild elk were tested for bTB in northwest Minnesota and all have been negative for the disease [15]. Natural predators included gray wolves (Canis lupus), black bears (Ursus americanus), coyotes (Canis latrans), red foxes (Vulpes vulpes), and bobcats (Felis rufus).
The $425-\mathrm{km}^{2}$-Bovine TB Core Area is located between $48^{\circ} 23^{\prime} \mathrm{N}$ and $48^{\circ} 35^{\prime} \mathrm{N}$ latitude and $95^{\circ} 24^{\prime} \mathrm{W}$ and $95^{\circ} 41^{\prime} \mathrm{W}$ longitude and lies within the Bovine TB Management Zone. Its boundaries were defined by a $3.2-\mathrm{km}$ buffer around all the bTB-infected deer found by fall 2006. Land ownership was approximately $56 \%$ public (state-owned), $41 \%$ private, and $3 \%$ tribal. Deer densities were estimated at $1.8-2.5 \mathrm{deer} / \mathrm{km}^{2}$ in January 2007, and following 3 consecutive winters of aggressive deer removal efforts were reduced to $0.7-$ $1.3 \mathrm{deer} / \mathrm{km}^{2}$ in January 2010. Eight of the $12 \mathrm{bTB}$-infected cattle herds were found within the bTB Core Area.

2.2. Bovine Tuberculosis Surveillance Methods. Hunter-harvested surveillance was used to estimate apparent prevalence of bTB in the local deer population. Initial sampling goals were designed to have $95 \%$ confidence of detecting the disease at a prevalence $\geq 1.0 \%$ (2005-2007). Surveillance was conducted within 25-km-radius circles around known bTBinfected cattle farms. However, when Minnesota was granted Split-state Status in 2008, sampling goals were dictated by USDA, and we expanded the surveillance area to the entire Split-state Zone. Sampling requirements were set at 1,500 deer from within the Zone and 300 deer outside the Zone (2008 and 2009). In 2010, the MNDNR successfully renegotiated a reduction in sampling requirements with USDA and focused sampling intensity (with $99 \%$ confidence of detecting the disease if prevalence was $\geq 0.5 \%$ ) within the Split-state Zone only.

In Minnesota, it is mandatory for all licensed hunters (big game only) to register their game. Hunters that registered their deer within the bTB Surveillance Area were asked to voluntarily submit cranial lymph node samples for bTB testing at designated registration stations. Only deer $>1.0$ year old were included in sampling efforts. Hunter information was recorded, including the hunter's name, address, telephone number, MNDNR number, and location of kill. Maps were provided to assist the hunters in identifying the location (Township, Range, Section, and Quarter-section) of the kill. Cooperating hunters were offered incentives, including a cooperator's patch and raffles for firearms donated by local and statewide sporting groups.

Additionally, MNDNR attempted to further reduce deer numbers in the posthunting season in the Bovine TB Core Area through the use of sharpshooters. The goal was to harvest as many deer as possible in hopes of removing additional bTB-infected deer from this critical area, thus, reducing the potential for deer-deer or deer-cattle transmission. Disease prevalence data generated from this removal effort were biased by targeting deer from known bTB-infected areas. During winters 2007-2010, sharpshooting from the ground was conducted by USDA-Wildlife Services (USDA-WS) professionals; supplemental sharpshooting was conducted by aerial operations during winters 2007 (Wildlife Services, Inc.) and 2008 (Tery Jon Aviation). Sharpshooter-harvested deer were transported intact to a central processing facility at Thief Lake Wildlife Management Area in northwest Minnesota. All deer were sampled, including fawns $(<1.0$ year old). Sample collection and handling is described below. 


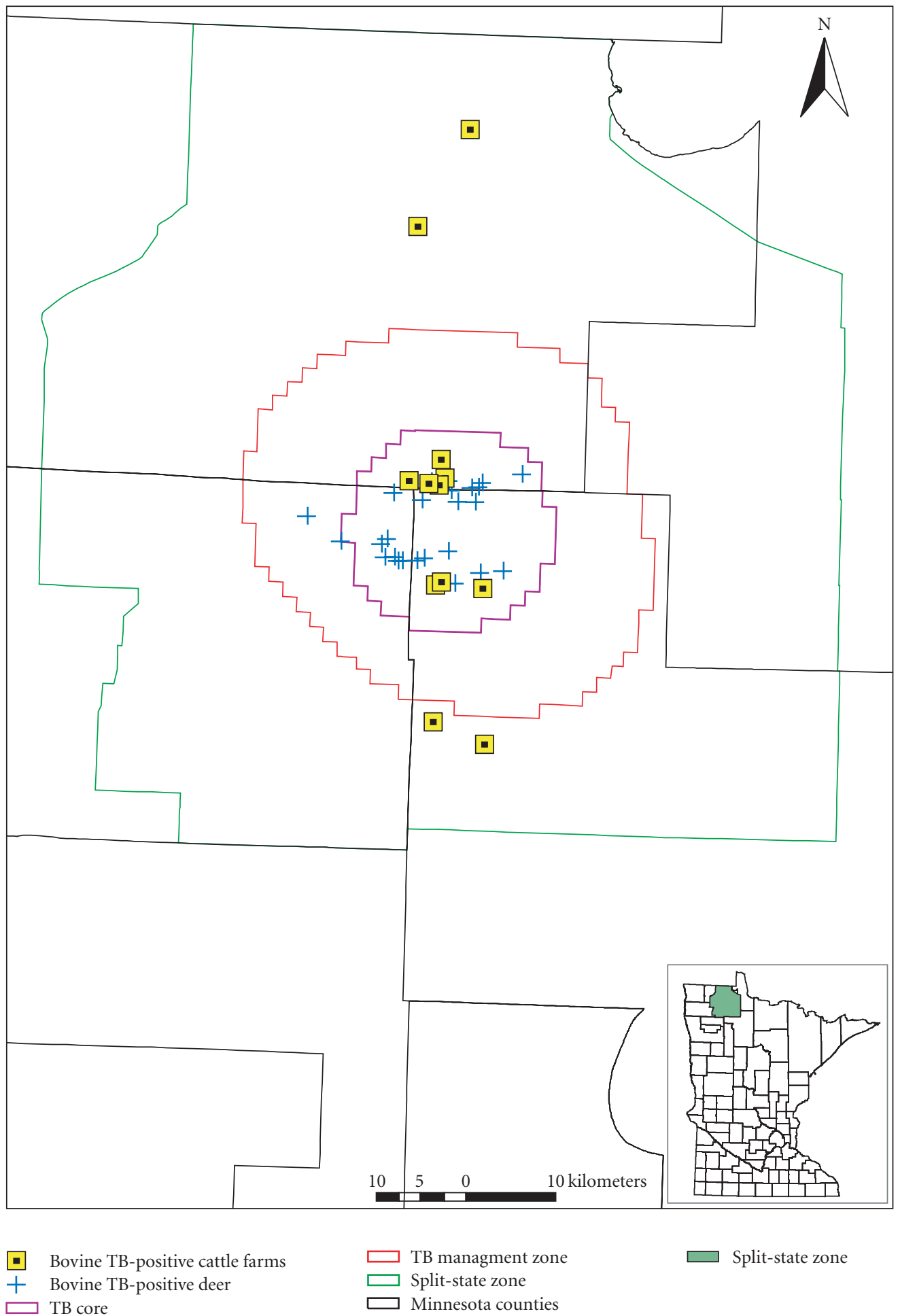

FIGURE 1: Spatial distribution of free-ranging white-tailed deer $(n=27)$ and cattle farms $(n=12)$ infected with bovine tuberculosis, 2005-2010, northwest Minnesota.

Carcasses that were free of any visible lesions were salvaged for venison and made available to the public.

2.3. Bovine Tuberculosis Sample Collection and Testing. Six cranial lymph nodes (parotid, submandibular, and medial retropharyngeal) extracted from all deer by trained personnel were submitted for testing, regardless of surveillance method. Once extracted, all lymph nodes were crosssectioned for presence of gross lesions. Tissue collection procedures included a visual inspection of the chest cavity of 
the hunter-killed deer (lungs were typically not available as carcasses were field-dressed). Any suspect carcasses (e.g., obvious lesions in chest cavity or lymph nodes) were confiscated at the registration stations, and the hunter was issued a replacement deer license at no charge. A first incisor was collected for aging by cementum annuli [16]. Suspect carcasses were transported in their entirety to the Veterinary Diagnostic Laboratory (VDL) at the University of Minnesota for further testing. Samples collected from sharpshooterkilled deer, vehicle-killed deer within the bTB Management Zone, or deer harvested by landowner shooting permit were handled as described above.

All lymph node samples were first submitted to the VDL for histological examination and acid-fast staining, and then forwarded to the National Veterinary Services Laboratories (NVSL) in Ames, IA for bacterial culture. Real-time polymerase chain reaction (RT PCR) was used to detect the presence of $M$. tuberculosis complex DNA when obvious lesions were present, which was then followed by bacterial culture. Samples that did not have obvious lesions were pooled in groups of 5 deer and also cultured. Bacterial growth identified as $M$. bovis indicates the animal was infected with bTB. These cultures were submitted for further laboratory testing to compare similarities or differences with other cultures of $M$. bovis identified within Minnesota and strains identified in the reference collection at NVSL.

2.4. Estimating Deer Densities in Bovine Tuberculosis Core Area. Deer surveys were conducted in late January-early February 2007-2010 to estimate deer densities and monitor changes in the abundance and distribution of deer within the bTB Core Area relative to ongoing management actions. The target population was free-ranging white-tailed deer within the $425-\mathrm{km}^{2}$ bTB Core Area. We used an equal-probability, generalized random-tessellation, stratified sampling design [17], which generated a 2D spatially balanced sample. The sampling frame included 164 Public Land Survey sections with a sample size of 72 plots (sampling rate $=0.439$ ). Surveys were conducted by helicopter (Bell OH-58, Jet Ranger [2007-2008, 2010]; Enstrom 480B [2009]) with a pilot and 2 observers. Population estimates reflected the minimum numbers of deer present during the sampling interval, as estimates were not adjusted for detectability. Deer movements between sample plots were assumed to be minimal. Confidence intervals ( $95 \%$ CIs) were based on sampling variance only and did not include uncertainty associated with detectability or animal movements.

\section{Results}

3.1. Bovine Tuberculosis Prevalence and Geographic Distribution. From 2005 to 2010, 6,955 hunter-harvested deer were tested for bTB in northwestern MN, which included 12 infected deer (Table 1). Age class of hunter-harvested deer were $65 \%$ adults $(\geq 2.0$ years old) and $35 \%$ yearlings ( 1.0 to $<2.0$ years old). Annual estimates in apparent prevalence demonstrated a declining trend, from $0.53 \%$ in 2006 to $0.07 \%$ in 2009 (Table 1, Figure 2). Final test results are

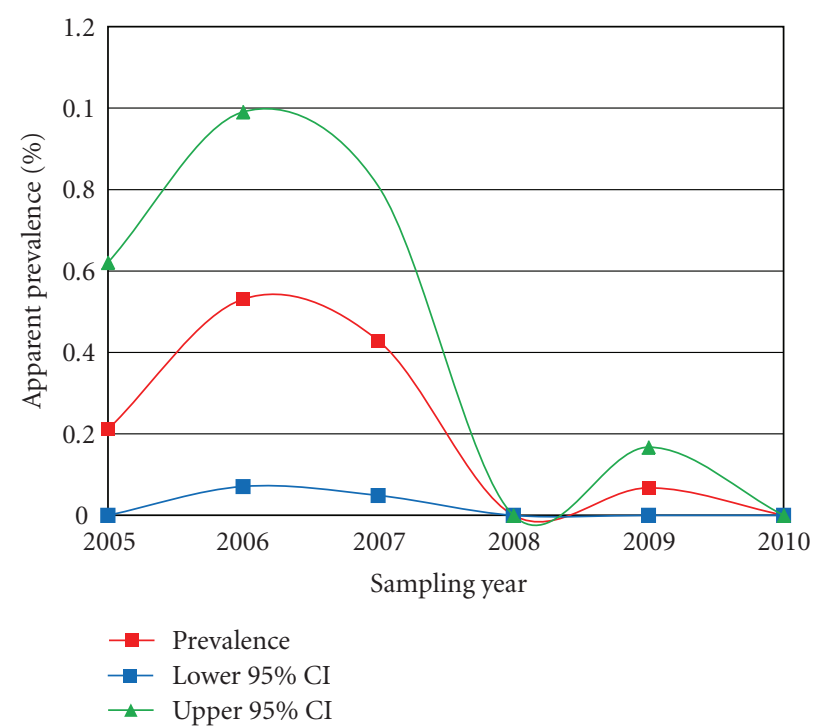

(a)

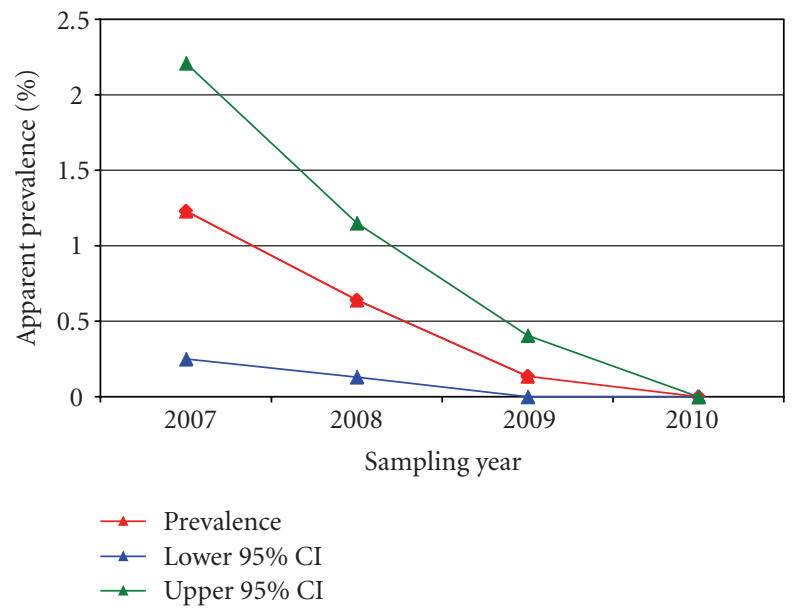

(b)

Figure 2: (a) Prevalence of bovine tuberculosis (bTB) in hunterharvested deer from 2005-2010 in the Bovine TB Surveillance Zone. (b) Prevalence of bTB from sharpshooter-removed deer from 20072010 in the Bovine TB Core Area, northwest Minnesota.

pending for fall 2010, but no obvious cases of bTB were identified through gross examinations. Also, 4,038 hunterharvested deer were tested outside of the northwest region during a one-time, statewide sampling effort in 2006; all deer were negative for bTB.

Sharpshooting removed 2,613 deer (22\% aerial gunning and $78 \%$ ground sharpshooting) from the bTB Core Area, including 14 infected deer (Table 1). Sharpshooter-harvested deer were $63 \%$ adults, $10 \%$ yearlings, and $27 \%$ fawns $(<1.0$ year old). Annual estimates indicated apparent prevalence declined from 1.23\% in 2007 to $0 \%$ in 2010 (Figure 2).

A total of 9,783 deer from all surveillance methods (2005-2010) were tested in northwest Minnesota for bTB and yielded 27 infected individuals (15 males, 12 females). All 27 bTB-infected deer were $>1.0$ year of age at harvest. Two deer harvested by sharpshooters in winter 2007 were 
TABLE 1: Number of deer sampled for bovine tuberculosis (bTB) and testing results listed by sampling strategy, 2005-2010, northwest Minnesota.

\begin{tabular}{|c|c|c|c|c|c|c|c|}
\hline \multirow{2}{*}{ Sampling strategy } & \multicolumn{7}{|c|}{ Surveillance year } \\
\hline & 2005 & 2006 & 2007 & 2008 & 2009 & 2010 & Totals \\
\hline Hunter-harvested (Oct-Jan) & 474 & 942 & 1,166 & 1,246 & 1,488 & 1,639 & 6,955 \\
\hline No. bTB-positive & 1 & 5 & 5 & 0 & 1 & 0 & \\
\hline Apparent prevalence (\%) & 0.21 & 0.53 & 0.43 & 0.00 & 0.07 & $0.00^{1}$ & \\
\hline Sharpshooting (Feb-April) & & & 488 & 937 & 738 & 450 & 2,613 \\
\hline No. bTB-positive & & & 6 & 6 & 2 & 0 & \\
\hline Apparent prevalence (\%) & & & 1.23 & 0.64 & 0.27 & 0.00 & \\
\hline Landowner shooting permit & & 90 & & 125 & & & 215 \\
\hline No. bTB-positive & & 1 & & 0 & & & \\
\hline Total no. deer tested & 474 & 1,032 & 1,654 & 2,308 & 2,226 & 2,089 & 9,783 \\
\hline Total no. bTB-positive & 1 & 6 & 11 & 6 & 3 & 0 & 27 \\
\hline
\end{tabular}

${ }^{1}$ Final culture results from fall 2010 hunter-harvested sampling are pending.

yearlings (1.5 years old). The remaining 25 infected deer were $>2.5$ years old. Twenty-six $(96 \%)$ of the infected deer were born on or before 2005. The 27th infected deer was 3.5 years old at harvest in fall 2009 (born in 2006). Twenty-one (76\%) of the bTB-infected deer presented with gross lesions inside the chest cavity (ribcage, lungs, and diaphragm) or cranial lymph nodes; these carcasses were confiscated. Three (10\%) of the bTB-infected deer presented with gross lesions in lymph nodes only (chest cavities were clear), and 4 (14\%) deer had microscopic lesions in the lymph nodes that were detected histologically.

The geographic distribution of bTB-infected deer was limited to a $16 \mathrm{~km}$ radius around the "index cattle herd," discovered in 2005 (Figure 1). Twenty-six of the bTBinfected deer were harvested within the boundaries of the bTB Core Area. The 27th infected deer (adult male harvested in fall 2009) was located $3.5 \mathrm{~km}$ outside of the western boundary of this area (Figure 1). Although this was the first case of a bTB-infected deer found outside the bTB Core, the distance was small enough that its homerange likely extended into the bTB Core Area. To be certain that this case did not represent geographic spread of the disease, winter sharpshooting efforts in 2010 were expanded to include $60 \mathrm{~km}^{2}$ to the west of the original bTB Core Area boundary. Approximately 200 deer were removed within a $3.5 \mathrm{~km}$ radius around this 27 th case and no additional bTB-infected deer were discovered.

3.2. Reducing Deer Densities in Critical Areas. Annual aerial surveys of the bTB Core Area estimated the deer population at $935( \pm 76, \mathrm{SE}), 807( \pm 75), 664( \pm 44)$, and $422( \pm 64)$ in winters 2007-2010, respectively. The deer population in the bTB Core declined 55\% from 2007 to 2010, following 3 consecutive years of winter sharpshooting and liberalized hunting opportunities (Figure 3 ).

Fall deer harvest in the special disease management unit, which encompassed the bTB Management Zone, registered $1,484,840,781$, and 765 deer during falls 2007-2010, respectively. Adults and yearlings comprised $75 \%$ of deer registrations with males ranging from $47 \%$ to $56 \%$; fawns accounted for the remaining $25 \%$ of the harvest.

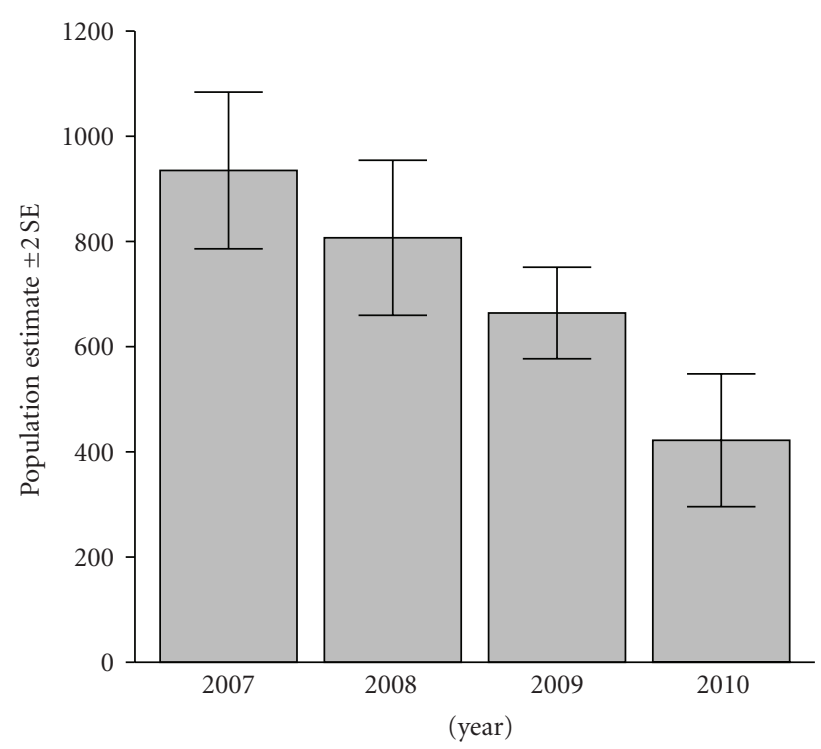

Figure 3: Population estimate of free-ranging white-tailed deer within the Bovine Tuberculosis Core Area, winters 2007-2010, northwest Minnesota.

3.3. Recreational Feeding of Deer. Recreational feeding of wild cervids was prohibited in a $10,060 \mathrm{~km}^{2}$ area of northwest Minnesota, which encompassed and was 6.5 times larger than the bTB Management Zone, in late November 2006. Enforcement flights identified 34 suspected illegal sites during winter 2007 within the bTB Management Zone. Officers visited the sites and offered verbal warnings $(n=$ $19)$, written warnings, $(n=3)$, or found no illegal activity $(n=12)$. In winter 2008 , enforcement efforts intensified for both illegal baiting and recreation feeding activities within the bTB Management Zone and 36 illegal sites were identified (19 baiting, 17 feeding). In these cases, officers issued verbal warnings $(n=2)$, written warnings $(n=3)$, citations $(n=12)$, or found no illegal activity $(n=19)$. Sixteen $(7$ baiting, 9 feeding) illegal sites were identified in winter 2009, leading to verbal warnings $(n=1)$, written warnings $(n=1)$, citations $(n=11)$, or no action $(n=3)$. In winter 2010, 
only 3 complaints ( 1 baiting and 2 feeding) were investigated, resulting in 2 citations and 1 unfounded report.

3.4. Risk Mitigation at the Wildlife-Cattle Interface. Wildlife risk assessments were conducted on 309 farms within the Split-State Zone in 2009, including 67 within the bTB Management Zone, as described in Knust et al. [18]. Locations of unprotected stored feed and cattle yards relative to available deer habitat were the primary factors elevating risk of disease transmission between cattle and deer. Within the bTB Management Zone, $48 \%$ of the farm risk assessments indicated moderate to high risk; $75 \%$ of this risk was attributed to feeding practices (including unprotected stored feed). Also, $83 \%$ of these farms had adequate daytime cover for deer, $43 \%$ reported deer on their farms during winter months, and $95 \%$ of the owners hunt deer on their land.

Following the cattle buy-out program in 2008, 34 fences were erected to protect stored feed and winter feeding sites throughout the bTB Management Zone. This amounted to $22,500 \mathrm{~m}$ of fencing. Compliance officers employed by BAH inspect these fences twice annually.

\section{Discussion}

When Minnesota first detected bTB in free-ranging deer, state wildlife and agricultural officials immediately contacted experts in Michigan, who have been battling a bTB outbreak in deer and cattle since 1995 [8, 19]. Many lessons could be learned from Michigan's efforts to control the disease, and their experience helped define Minnesota's approach to eradicating bTB in deer and cattle.

4.1. Rapid Response to Initial Detection. Through epidemiological investigations, Minnesota BAH and USDA concluded that bTB was likely introduced into cattle in the northwest 3-5 years prior to the initial detection by routine slaughter surveillance $[9,14]$. One major piece of evidence to support this conclusion was that herd prevalence was low among bTB-infected farms; it was $<2 \%$ and $5-6 \%$ in 8 and 3 herds, respectively. This meant only one cow was found infected in most of these herds. Only one herd, the 12th bTB-infected farm (Jan 2009), had a high herd prevalence (15\%) that included 3 infected cows and 9 calves (10 months old). Surprisingly, this 12 th herd had tested negative during 2 previous whole herd bTB tests. Also, all bTB-infected herds were depopulated within a few months of disease detection; the removal of infected animals likely helped reduce $M$. bovis exposure risks to both cattle and deer.

MNDNR responded immediately to the initial detection of bTB in cattle by initiating hunter-harvested surveillance in free-ranging deer in fall 2005. Just prior to the bTB outbreak, MNDNR had been conducting hunter-harvested surveillance for chronic wasting disease (2002-2004) and so had a protocol in place for obtaining biological samples from deer at registration stations. More importantly, the agency also had dedicated funding available through license dollars to support disease surveillance work. Being poised financially and logistically to respond to the bTB threat facilitated MNDNR's rapid response.

4.2. Follow-Through on Monitoring the Outbreak with Adequate Surveillance. When MNDNR discovered the first bTBinfected deer, its significance was questionable. It was harvested within $2 \mathrm{~km}$ of an infected cattle farm and was the same strain of bTB. On-farm shooting permits offered in early winter 2006 produced a second bTB-infected deer that, was again, directly and similarly linked to an infected cattle farm. Although this appeared to be a simple spillover event with limited infection in free-ranging wildlife, the Michigan example of bTB establishing a wildlife reservoir in freeranging deer concerned state and federal officials enough to warrant a geographically expanded surveillance effort in fall 2006.

Further, MNDNR decided to focus surveillance efforts on deer and not spend valuable resource dollars on evaluating infection in other wildlife species, such as furbearers or rodents. While furbearers are important wildlife reservoirs for bTB elsewhere in the world $[6,7]$, research conducted in Michigan on carnivores and scavengers concluded these species were only spill-over hosts that lacked the ability to effectively transmit the disease $[20,21]$. Atwood et al. [22] suggested coyotes may act as useful sentinels for bTB infection in cervids; however, Sangster et al. [23] concluded testing coyotes lacked sufficient sensitivity to be effective. Given the apparent lack of epidemiological importance of furbearers in bTB transmission between deer and cattle, MNDNR chose to focus surveillance efforts solely on cervids.

The importance of adequate and timely surveillance was also learned from Michigan's bTB outbreak. Michigan's first case of bTB in a hunter-killed deer was reported in 1975; yet no surveillance in wildlife was conducted until a second grossly lesioned deer surfaced in 1994. Surveillance was initiated in 1995 within a $16 \mathrm{~km}$ radius of this case, and a $5 \%$ apparent prevalence of bTB was discovered [19]. This high initial prevalence suggested that bTB was maintaining itself in this deer herd for at least 2 decades before being discovered. By contrast, Minnesota's initial prevalence was only $0.2 \%$.

4.3. Recognize When Monitoring Must Switch to Management. The discovery of 5 additional bTB-infected deer, combined with more cattle infection and the loss of Minnesota's bTB-free accreditation, triggered a switch in MNDNR's approach to the disease outbreak. No longer were officials debating over the significance of the 2 initial deer detected in direct association with infected cattle farms. Although spillover from cattle was the likely origin of infection, the fact remained that bTB was present in wild deer, and the possibility of establishing a disease reservoir in deer needed to be addressed. MNDNR decided to develop additional methods to reduce deer numbers and infected animals, to prevent or slow the establishment of a bTB reservoir in deer.

Public support, including the cattle industry and sporting groups, was needed to effectively launch an aggressive disease control program. The loss of bTB-Free accreditation 
and cattle industry pressure drove BAH to attempt to swiftly contain the outbreak in cattle and provide assurances to trading partners that disease control efforts were effective. However, the MNDNR was not operating under any regulatory framework nor was any financial support (state or federal) offered to manage the outbreak in deer. With the Michigan example in mind, the MNDNR recognized that the wildlife component of this outbreak would play a vital role in the state's efforts to regain its bTB accreditation and committed to the shared goal with BAH to eradicate the disease as soon as possible.

Establishing a dual-agency approach was challenging, as key stakeholder groups had differing priorities and ideas on how bTB should be managed in the state. Yet, a collaborative approach was launched, with shared messages of the importance of this disease to both the cattle industry and deer hunting community. Joint outreach efforts were initiated, including local media articles, public meetings, and websites, to inform the local community in northwest Minnesota of imminent changes in both deer management and cattle regulations. Local ad hoc groups formed, including a bTB Task Force, to help communicate local issues concerning bTB management to state and federal agencies. Political champions were also identified by industry groups to help fund management efforts and provide support for aggressive actions that would follow.

4.4. Aggressively Reduce Transmission Potential. Results of 5 years of bTB surveillance in deer have demonstrated that disease prevalence has been reduced to an undetectable level and the geographic extent of infected individuals is limited. Although apparent prevalence in 2005 was less than 2006 $(0.21 \%$ versus $0.53 \%)$, it is unlikely that an increase in infection truly existed in the deer population between years. It is probable that the sample collected in fall 2005 was not sufficiently large and underestimated apparent prevalence. We are confident that our surveillance has been effective in identifying bTB-infected deer, as $85 \%$ of the positive cases were detected through gross examination of tissues alone. Yet every deer sampled was submitted for bacterial culture; thus our apparent prevalence estimates may accurately reflect true disease prevalence of bTB in the local deer population [24]. Michigan's surveillance program only submits grossly lesioned individuals for bacterial culture to reduce testing costs, and O'Brien et al. [24] reported this sampling method underestimated true prevalence by about $25 \%$.

Age structure of the bTB-positive deer demonstrated a lack of infection in younger age classes. This is important because yearling prevalence has been used as a crude index of the rate of new infections, as well as evidence that deerto-deer transmission is occurring [25]. The absence of bTB infection in younger deer strongly suggests that this disease was not maintaining itself in Minnesota's deer population. Our "Alive in ' 05 " theory accurately predicted the birth year of 26 of the 27 bTB-infected deer as on or before 2005, when the disease was first detected and most prevalent in cattle. Assuming the disease spilled over from bTB-infected cattle just prior to 2005, and that deer-deer transmission was absent or minimal, we would expect only this older age cohort of deer to be infected. The only outlier to this theory was the 27th bTB-infected deer born in 2006; however, this deer could have been exposed to the remaining infected cattle herds that were discovered in 2006-2009 or was an isolated occurrence of deer-deer transmission. Also, epidemiological modeling in Michigan has demonstrated a sex-based bias in infected deer, with adult males more likely to have bTB than adult females [25]. We did not observe a sex-based difference in infection rates, as both sexes were equally represented in bTB-infected individuals.

Our aerial survey data indicated a $55 \%$ reduction in deer densities within the bTB Core Area. During winters 2008-2010, the number of deer harvested by sharpshooting exceeded the early winter population estimates. While it is, of course, not possible that deer removal efforts killed $>100 \%$ of the deer in the bTB Core Area, it is likely that deer were continually moving in and out of this area. There was no physical barrier to prevent movement in or out of the bTB Core Area, but immigration and emigration rates are unknown. Further, deer surveys estimated the deer population at one point in time (typically late Jan or early Feb), while the sharpshooting efforts began in late February and continued through April. It is likely that deer migration from winter range to spring-summer-fall ranges accounted for the increased movement activity noted in April, although migration rates are also poorly understood for this portion of the state.

While fall deer harvest likely contributed to the reduction of deer within the bTB Core Area, total registration within the special disease management unit dropped nearly $50 \%$ from 2007 to 2008, and remained at that reduced level through 2010. This is likely due to fewer deer being available to hunters because of winter sharpshooting. Hunters also perceived a negative effect of winter sharpshooting on deer available to harvest, thus hunting pressure was reduced [26]. Increased hunting opportunities, including special seasons and low cost bonus tags, did not effectively increase deer harvest. This was similar to experiences reported in other states, including Michigan, Wisconsin, and Illinois, where hunting opportunities were liberalized to encourage increased deer harvest for disease management $[8,27]$.

Efforts to restrict recreational feeding have been very successful. Since the ban were instituted in 2006, reports of illegal feeding activities have dropped markedly. Concurrent efforts to increase enforcement of baiting regulations likely increased compliance with feeding restrictions as well. In Michigan, regulatory efforts to restrict feeding and baiting have met with mixed success [28]. Much of the large-scale feeding activities there have subsided, but some smaller, covert feeding activities persist [8].

On the cattle side, the main action that dramatically reduced bTB transmission potential was the cattle buy-out program. A monumental effort to obtain adequate funding and producer buy-in to accept this voluntary program and remove 6,200 cattle from the bTB Management Zone. This $\$ 4.6$ million dollar program offered the farmer $\$ 500$ in addition to the slaughter value of each cow, and a $\$ 75 /$ head annual stipend to remain out of the cattle business until the 
state regains its bTB-free accreditation. Further, a 10: 90 cost share was provided to farmers to erect deer exclusion fencing around stored feed or cattle feeding areas up to a maximum of $\$ 75,000$. In 2009,34 fences equating to $22,500 \mathrm{~m}$ of fencing material were installed to mitigate risk of disease between cattle and wildlife, at a cost of $\$ 815,000$ to the state. Wildlife risk assessments were also conducted on all farms within the Split-state Zone [29]. Although these risk assessment were useful tools to educate farmers on practices that can mitigate risk of disease transmission between cattle and wildlife, it provided recommendations, but did not impose requirements. The only required risk mitigation practices apply to cattle farmers that receive state-sponsored fencing, where compliance is enforced and fences are inspected twice annually.

4.5. Evaluation of Efforts and Adjusting As Needed. Thus far, efforts to eradicate bTB in Minnesota have been costly. The USDA has spent nearly $\$ 70$ million, BAH $\$ 12.5$ million, and MNDNR \$3.5 million on bTB-related expenditures [29]. The average costs for hunter-harvested deer surveillance were $\$ 350,000 /$ year and sharpshooting ranged from $\$ 250,000$ to $\$ 600,000 /$ year, depending on whether aerial gunning was used to supplement ground removal efforts.

When MNDNR began sharpshooting deer, the public accepted the action as necessary to eradicate the disease in deer, but it was not popular. However, as the sharpshooting program persisted through 4 consecutive winters, and fewer bTB-infected deer were found each year, public tolerance declined markedly. This was of particular concern, because our primary method for disease surveillance was through hunter-harvested sampling, a voluntary process that required the cooperation of hunters. A loss of public support for MNDNR's management of bTB in deer could have made disease monitoring notably more challenging. In 2010, MNDNR was able to successfully negotiate with USDA for a reduced sampling requirement, which meant surveillance goals could be achieved through hunter-harvested surveillance alone, without the need for sharpshooting to augment sample size. Public distaste for sharpshooting motivated hunters to increase their participation in fall bTB surveillance. In fall 2010, hunters provided over $1.5 \mathrm{x}$ the required sample needed to satisfy the bTB sampling requirements. With sampling requirements met and no obvious cases of bTB evident in the hunter-harvested deer, no sharpshooting occurred in winter 2011.

Given all the aggressive management efforts that have occurred, did we effectively reduce bTB transmission potential? Substantial reductions in both deer densities and cattle numbers occurred from 2005 to 2010 . Recreational feeding and baiting activities have declined and separation of cattle and deer on farms has improved through enhanced risk mitigation practices. Further, ongoing bTB surveillance of deer has demonstrated a decline in disease prevalence and no geographic spread of infected individuals. Bovine TB has not been found in a cattle herd since January 2009, with over a year of continued testing of herds. These results are encouraging, yet we must be cautious about prematurely pronouncing bTB eradicated in Minnesota. Continued surveillance is needed to confirm the absence of this disease in Minnesota's deer.

\section{Conclusions}

Minnesota's approach to bTB control employed the same basic intervention strategies as Michigan, namely, reducing transmission potential by reducing deer densities and restricting feed/baiting activities. Yet results have been very different. When active disease control was initiated in Michigan, deer were likely already a bTB reservoir, but in Minnesota, deer were likely spillover hosts. Minnesota had other key advantages from the onset of the disease outbreak. Deer densities were 6-8 times less than in Michigan, and Minnesota's bTB Core was 3 times smaller in size. Further, $60 \%$ of Minnesota's bTB Core was publically owned which facilitated aggressive deer removal efforts; Michigan's core area is only $10 \%$ public. Baiting and feeding of deer in Michigan persist, despite regulations; whereas these activities are under control in Minnesota. The MNDNR also had adequate dedicated funding in hand for initial disease management needs. Finally, public tolerance can exert a significant effect on potential disease management strategies, and thus the overall success of control efforts [30]. Minnesota was fortunate to have support from the public, industry, sporting groups, and key political champions to aggressively manage the bTB outbreak.

\section{Acknowledgments}

The authors would like to thank the Minnesota Department of Natural Resources, Division of Fish \& Wildlife, for the financial, logistical, and staff support for bovine tuberculosis management. Financial support was also received from the Minnesota State Legislature and USDA-APHIS-Veterinary Services. USDA-APHIS-Wildlife Services did an outstanding job on winter deer removal activities, and they would like to thank the National Wildlife Disease Program for providing wildlife disease biologist that assisted with sampling efforts. They also thank the Minnesota Deer Hunter's Association for their support.

\section{References}

[1] R. S. Morris, D. U. Pfeiffer, and R. Jackson, “The epidemiology of Mycobacterium bovis infections," Veterinary Microbiology, vol. 40, no. 1-2, pp. 153-177, 1994.

[2] L. A. L. Corner, "The role of wild animal populations in the epidemiology of tuberculosis in domestic animals: how to assess the risk," Veterinary Microbiology, vol. 112, no. 2-4, pp. 303-312, 2006.

[3] V. Naranjo, C. Gortazar, J. Vicente, and J. de la Fuente, "Evidence of the role of European wild boar as a reservoir of Mycobacterium tuberculosis complex," Veterinary Microbiology, vol. 127, no. 1-2, pp. 1-9, 2008.

[4] V. De Vos, R. G. Bengis, N. P. J. Kriek et al., “The epidemiology of tuberculosis in free-ranging African buffalo (Syncerus caf$\mathrm{fer}$ ) in the Kruger national park, South Africa," Onderstepoort Journal of Veterinary Research, vol. 68, no. 2, pp. 119-130, 2001. 
[5] J. S. Nishi, T. Shury, and B. T. Elkin, "Wildlife reservoirs for bovine tuberculosis (Mycobacterium bovis) in Canada: strategies for management and research," Veterinary Microbiology, vol. 112, no. 2-4, pp. 325-338, 2006.

[6] R. J. Delahay, A. N. S. De Leeuw, A. M. Barlow, R. S. CliftonHadley, and C. L. Cheeseman, "The status of Mycobacterium bovis infection in UK wild mammals: a review," The Veterinary Journal, vol. 164, no. 2, pp. 90-105, 2002.

[7] J. D. Coleman and M. M. Cooke, "Mycobacterium bovis infection in wildlife in New Zealand," Tuberculosis, vol. 81, no. 3, pp. 191-202, 2001.

[8] D. J. O’Brien, S. M. Schmitt, S. D. Fitzgerald, D. E. Berry, and G. J. Hickling, "Managing the wildlife reservoir of Mycobacterium bovis: the Michigan, USA, experience," Veterinary Microbiology, vol. 112, no. 2-4, pp. 313-323, 2006.

[9] L. Glaser, Minnesota's Strategy for Eliminating Bovine Tuberculosis, Minnesota Board of Animal Health, St. Paul, Minn, USA, 2008.

[10] B. Harris, Mycobacterium Bovis Genotyping Report, National Veterinary Services Laboratory, Ames, Iowa, USA, 2009.

[11] M. Carstensen, D. Pauly, M. W. DonCarlos, and L. Cornicelli, "Managing bovine tuberculosis in free-ranging white-tailed deer in northwestern Minnesota: a 2007 progress report," in Summaries of Wildlife Research Findings 2007, pp. 1-9, Minnesota Department of Natural Resources, Wildlife Population and Research Unit, St. Paul, Minn, USA, 2008.

[12] United States Department of Agriculture, Bovine Tuberculosis Eradication: Uniform Methods and Rules, Effective, January 2005, http://www.aphis.usda.gov/animal_health/animal_diseases/tuberculosis/downloads/tb-umr.pdf.

[13] M. V. Palmer and D. L. Whipple, "Survival of Mycobacterium bovis on feedstuffs commonly used as supplemental feed for white-tailed deer (Odocoileus virginianus)," Journal of Wildlife Diseases, vol. 42, no. 4, pp. 853-858, 2006.

[14] L. Glaser, Minnesota Bovine Tuberculosis Eradication Program: Application for Split-State Status, Minnesota Board of Animal Health, St. Paul, Minn, USA, 2008.

[15] E. H. Hildebrand, M. Carstensen, E. Butler, and L. Cornicelli, "Preliminary results from herd health assessment for northwestern free-ranging elk from 2004-2009," in Summaries of Wildlife Research Findings 2009, pp. 135-149, Minnesota Department of Natural Resources, Wildlife Population and Research Unit, St. Paul, Minn, USA, 2010.

[16] F. F. Gilbert, "Aging white-tailed deer by annuli in the cementum of the first incisor," Journal of Wildlife Management, vol. 30, no. 1, pp. 200-202, 30.

[17] D. L. Stevens and A. R. Olsen, "Spatially balanced sampling of natural resources," Journal of the American Statistical Association, vol. 99, no. 465, pp. 262-278, 2004.

[18] B. Knust, P. C. Wolf, and S. Wells, "Use of an on-farm assessment to characterize risk," American Journal of Veterinary Research. In press.

[19] S. M. Schmitt, S. D. Fitzgerald, T. M. Cooley et al., "Bovine tuberculosis in free-ranging white-tailed deer from Michigan," Journal of Wildlife Diseases, vol. 33, no. 4, pp. 749-758, 1997.

[20] C. S. Bruning-Fann, S. M. Schmitt, S. D. Fitzgerald et al., "Bovine tuberculosis in free-ranging carnivores from Michigan," Journal of Wildlife Diseases, vol. 37, no. 1, pp. 5864, 2001.

[21] T. C. Atwood, T. J. Deliberto, H. J. Smith, J. S. Stevenson, and K. C. Vercauteren, "Spatial ecology of raccoons related to cattle and bovine tuberculosis in Northeastern Michigan," Journal of Wildlife Management, vol. 73, no. 5, pp. 647-654, 2009.
[22] T. C. Atwood, K. C. Vercauteren, T. J. Deliberto, H. J. Smith, and J. S. Stevenson, "Coyotes as sentinels for monitoring bovine tuberculosis prevalence in white-tailed deer," Journal of Wildlife Management, vol. 71, no. 5, pp. 1545-1554, 2007.

[23] C. Sangster, D. Bergeson, C. Lutze-Wallace, V. Crichton, and G. Wobeser, "Feasibility of using coyotes (Canis latrans) as sentinels for bovine mycobacteriosis (Mycobacterium bovis) infection in wild cervids in and around riding Mountain National Park, Manitoba, Canada," Journal of Wildlife Diseases, vol. 43, no. 3, pp. 432-438, 2007.

[24] D. J. O’Brien, S. M. Schmitt, D. E. Berry et al., "Estimating the true prevalence of Mycobacterium bovis in hunter-harvested white-tailed deer in Michigan," Journal of Wildlife Diseases, vol. 40, no. 1, pp. 42-52, 2004.

[25] D. J. O’Brien, S. M. Schmitt, J. S. Fierke et al., "Epidemiology of Mycobacterium bovis in free-ranging white-tailed deer, Michigan, USA, 1995-2000," Preventive Veterinary Medicine, vol. 54, no. 1, pp. 47-63, 2002.

[26] M. Schermann and S. Wells, Bovine Tuberculosis Transmission and Control Initiative: Final Report, Center for Animal Health and Food Safety, University of Minnesota, St. Paul, Minn, USA, 2010.

[27] T. R. Van Deelen and D. R. Etter, "Effort and the functional response of deer hunters," Human Dimensions of Wildlife, vol. 8, pp. 97-108, 2003.

[28] B. A. Rudolph, S. J. Riley, G. J. Hickling, B. J. Frawley, M. S. Garner, and S. R. Winterstein, "Regulating hunter baiting for white-tailed deer in Michigan: biological and social considerations," Wildlife Society Bulletin, vol. 34, no. 2, pp. 314-321, 2006.

[29] B. Thompson, Minnesota's Bovine Tuberculosis Management Plan, Minnesota Board of Animal Health, St. Paul, Minn, USA, 2010.

[30] M. Carstensen, D. J. O’Brien, and S. M. Schmitt, "Public acceptance as a determinant of management strategies for bovine tuberculosis in free-ranging U.S. wildlife," Veterinary Microbiology. In press. 

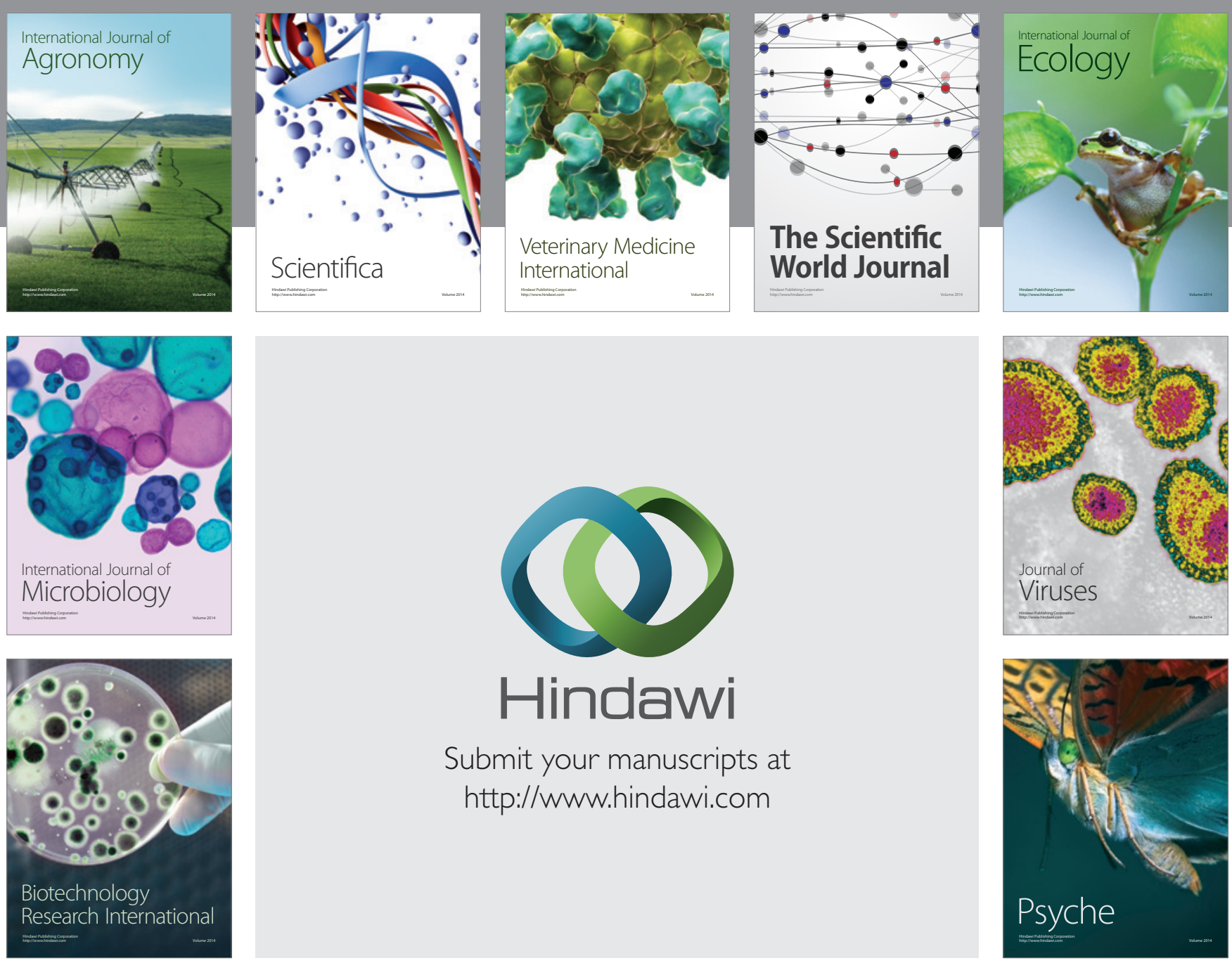

Submit your manuscripts at

http://www.hindawi.com
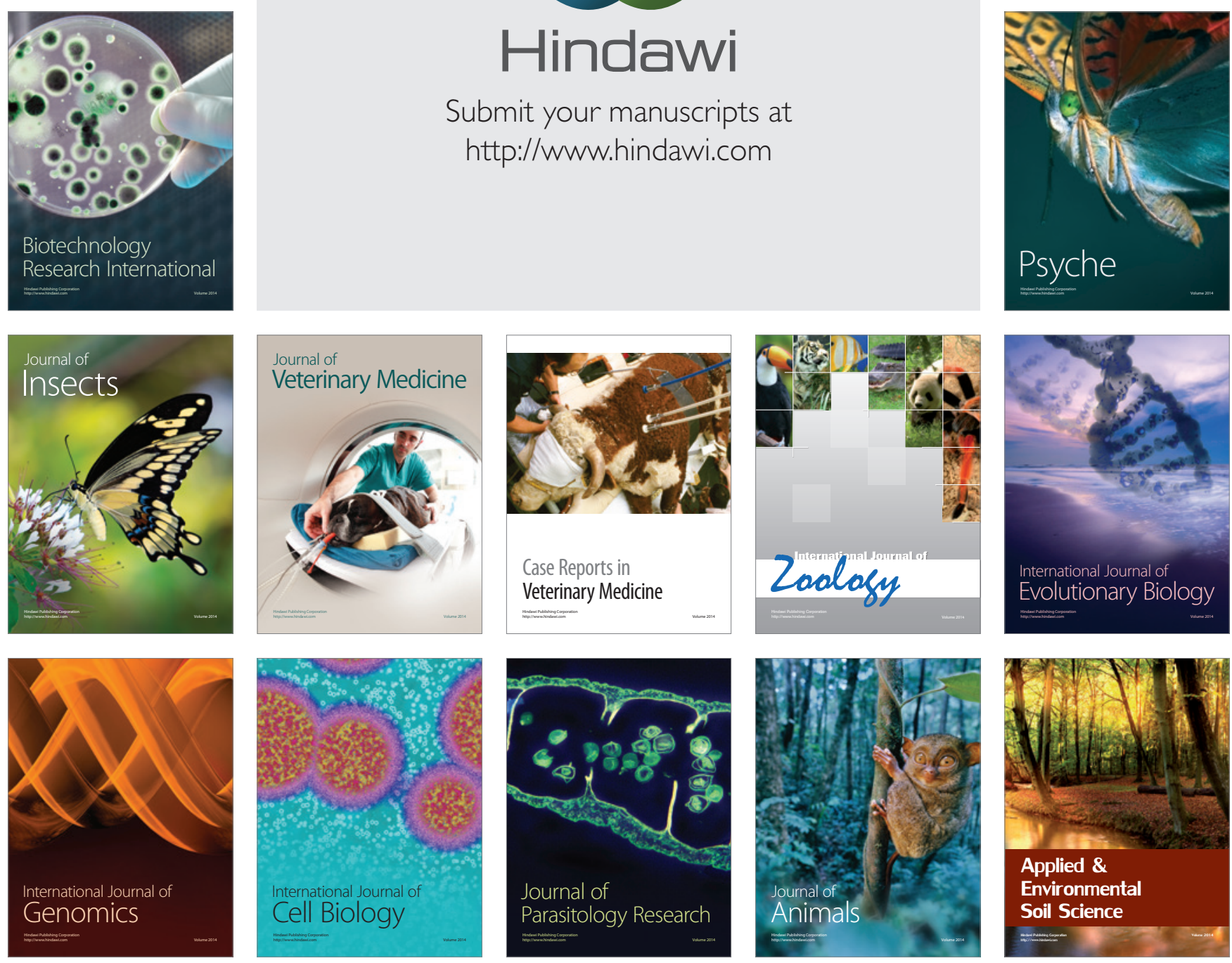